\title{
Finite element simulation and experimental investigation of cold forward extrusion process
}

\author{
Dorin Luca* \\ Gheorghe Asachi Technical University of Iasi, Department of Technologies and Equipment for \\ Materials Processing, Prof.dr.doc. D. Mangeron 51, 700050 Iasi, Romania
}

\begin{abstract}
Extrusion is the plastic deformation process that allows for the highest degree of complexity profiles to be obtained. This paper presents the simulation of a cold forward extrusion process using the finite element method. The results obtained show the stresses, strains and temperatures during the plastic deformation of the material, as well as the stresses and strains in the punch and die. The analysis of the results obtained for different geometric dimensions of the working tools allowed the optimization of the studied extrusion process. In order to validate the finite element model, experiments were carried out with the data acquisition from the real process, which allowed the appreciation that numerical and experimental data are found in a good agreement.
\end{abstract}

\section{Introduction}

Extrusion is applied both as a primary process and as a secondary manufacturing process. As a primary process, extrusion is carried out hot and by means of horizontal hydraulic presses with low deformation rates using rolled or cast semi-finished products whose cross-sectional dimensions reach $150 \ldots 500 \mathrm{~mm}$. As a secondary process, it is generally carried out cold, by means of mechanical presses with high deformation velocities, using semi-finished products of relatively small size.

The most important advantages of the extrusion procedure are: obtaining wide degree of deformation which ensures high productivity; obtaining complex configuration profiles that cannot be achieved by other plastic deformation processes; obtaining parts with dimensional accuracy and high surface quality; obtaining parts of materials that are difficult to process by other plastic deformation processes due to the presence of compressive stresses in the deformation zone.

The production of extruded products of good quality requires knowledge of influence factors, such as: stress state in the deformation zone; the flow pattern of the material; resistance to deformation of the material; friction between tools and material; shape and geometry of dies, etc. All these factors and other issues are the subject of an ongoing research on a global scale, with the aim of better capitalization of the materials, obtaining extruded parts with improved mechanical properties, extrusion of metallic materials with low plasticity.

\footnotetext{
*Corresponding author: dluca@tuiasi.ro
} 
A new manufacturing method, called friction extrusion process [1], is used to produce metal wires from metal powders or chips recycled without melting. The authors developed a mathematical model of the friction extrusion process that takes into account the heat transfer, material flow and then performed numerical simulations of the extrusion process. Another new method of forward extrusion with a reversible rotating die is presented in the paper [2]. The authors investigated the microstructure and properties of the obtained zinc parts, which they compared with the microstructure and properties of conventional extruded parts. A significant increase in the properties of rotary die extruded parts has been observed over conventional extrusion parts.

The microstructure and mechanical properties evaluation of the AZ91 magnesium alloy tubes produced by the radial-forward extrusion method was made in the paper [3]. Tubes produced by radial-forward extrusion present high strength and fine grained. Finite element simulation was applied to visualize effective strain at various deformation steps, variation of strain rate according to punch displacement, and force variation versus to the press ram's stroke.

The influence of the various deformation paths on the mechanical properties of the finished parts obtained of chips by hot extrusion was investigated in the paper [4]. Two hot extrusion methods have been studied: forward extrusion to obtain different types of shafts and backward extrusion to produce cans with different wall thicknesses. The influence of the strain path on the texture and microstructure is studied in an extrusion process of $\mathrm{Mg}$ $1 \mathrm{wt} \% \mathrm{Al}$ round bar [5]. The variation of texture within the extruded round bar determines the heterogeneity of its mechanical properties.

The numerical analysis methods of extrusion processes frequently used are upper bound method and finite element method (FEM).

In the paper [6] was investigated the forward extrusion process of an L-shaped, rectangular, hexagonal sections and spur gears. The upper bound method has been used to determine the velocity and strain distribution, the three-dimensional flow of the material and process loading. The comparison of the analytical results obtained with the upper bound method was done with numerical data obtained with the finite element method.

Simulation by FEM was used to determine energy consumption in forward and backward extrusion of a round section bar [7], to determine friction by a combined forward and backward extrusion test [8], to investigate plastic flow in a forward-backward-radial extrusion process [9], to investigate the lubricating effect of forward extrusion of an AA6063 aluminum alloy [10] or to establish the dependence between damage and the properties of the material in a hybrid forward extrusion process [11]. When performing the finite element analysis, the right choice of software tools [12], the number and type of finite elements, constraints and loads, etc., must be given high importance, so that the numerical data is to be in agreement with the experimental data.

Friction and wear extrusion tools are elements that must be considered for increasing the life of tools and avoidance of their failure.

This paper presents a case study for cold forward extrusion of a round section bar. The study presents a finite element analysis, performed in order to determine the influence of deformation cone angle of the die on extrusion force and stresses into the extrusion tools to optimize the studied process.

\section{Finite element simulation}

A finite element simulation of a cold forward extrusion process of a round section bar is presented (Figure 1). Deformation cone angle of the die plays an important role in achieving a proper flow of the material. Also, deformation cone angle of die value have a great influence on: extrusion load, extrusion energy and dies durability. 
(a)

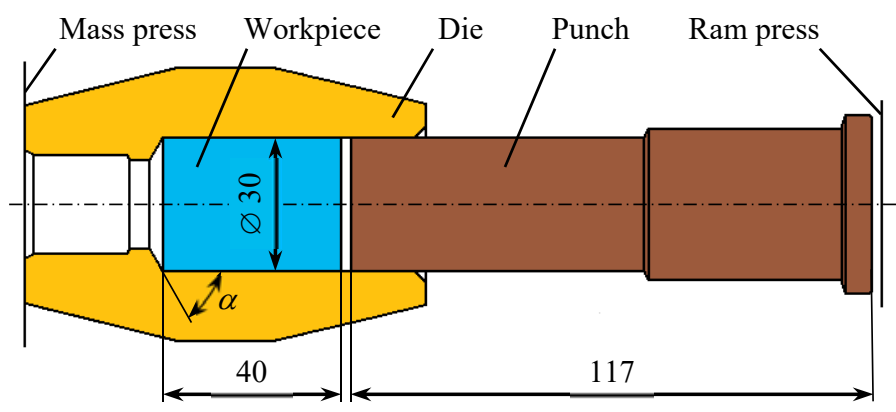

(b)

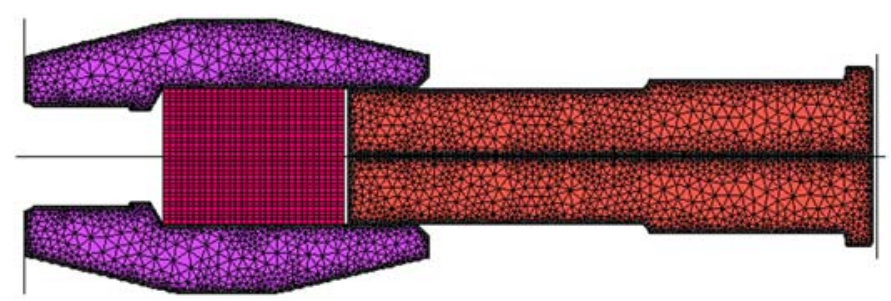

Fig. 1. Experimental extrusion system scheme (a) and 2D finite element model (b).

The chosen type of finite element analysis was coupled elasto-plastic, which enables establishing the dependency between stress and the temperature from the process. Coupled analysis considers the effect of temperature changes on the deformed material properties. Extrusion process occurs with the transfer of heat to the environment, being considered even the heat resulting from friction between the tools and workpiece, which is calculated based on the updated shape (of workpiece) after each increment and contact conditions. A fraction of the energy used for plastic deformation is converted into heat, and the friction between the tools and the workpiece also produces heat. Because of the axial-symmetric geometry of the extruded part, the simulation can be more efficient in terms of time analysis by adopting a 2D model analysis.

The workpiece was defined as a deformable body, was meshed by a number of 450 quadrilateral finite elements and an adaptive remeshing method was selected. The material model used is the elasto-plastic with hardening, material is assumed to be isotropic and von Mises plasticity criterion was used in the analysis. Both tools, die and punch, were defined as deformable bodies and meshed with triangular finite elements. Material data for the workpiece (lead) used in experiment, and for tools (15CrNi6 steel) are shown in Table 1.

Table 1. Material data for workpiece and tools.

\begin{tabular}{|l|c|c|l|c|c|}
\hline $\begin{array}{c}\text { Mechanical } \\
\text { characteristics }\end{array}$ & $\begin{array}{c}\text { Work- } \\
\text { piece }\end{array}$ & Tools & \multicolumn{1}{|c|}{$\begin{array}{c}\text { Thermal } \\
\text { characteristics }\end{array}$} & $\begin{array}{c}\text { Work- } \\
\text { piece }\end{array}$ & Tools \\
\hline Young's modulus $[\mathrm{MPa}]$ & 14000 & 200000 & Thermal expansion $[1 / \mathrm{K}]$ & $29.3 \times 10^{-6}$ & $10.0 \times 10^{-6}$ \\
\hline Poisson's ratio & 0.44 & 0.30 & Conductivity $[\mathrm{W} / \mathrm{m} \cdot \mathrm{K}]$ & 31 & 25 \\
\hline Mass density $\left[\mathrm{kg} / \mathrm{m}^{3}\right]$ & 11340 & 7700 & Specific heat $[\mathrm{J} / \mathrm{kg} \cdot \mathrm{K}]$ & 130 & 460 \\
\hline
\end{tabular}

The initial temperature for workpiece and tools at the start of the forward extrusion process was $20^{\circ} \mathrm{C}$. Contact conditions were established by specifying the coefficient of heat transfer with the environment for the workpiece, the coefficient of contact heat transfer and the initial temperature for tools and the coefficient of friction between the tools and the workpiece. The friction coefficient between the tools and the workpiece is assumed to be constant along the contact surfaces $(\mu=0.3)$. 


\section{Results and discussion}

The first results and analyzes undertaken followed the flow behavior of the material and the variations strains, stresses and temperature in the workpiece volume during the extrusion. Some results regarding the equivalent plastic strain, equivalent stress and temperature fields from the extruded part are graphically illustrated in Figure 2.
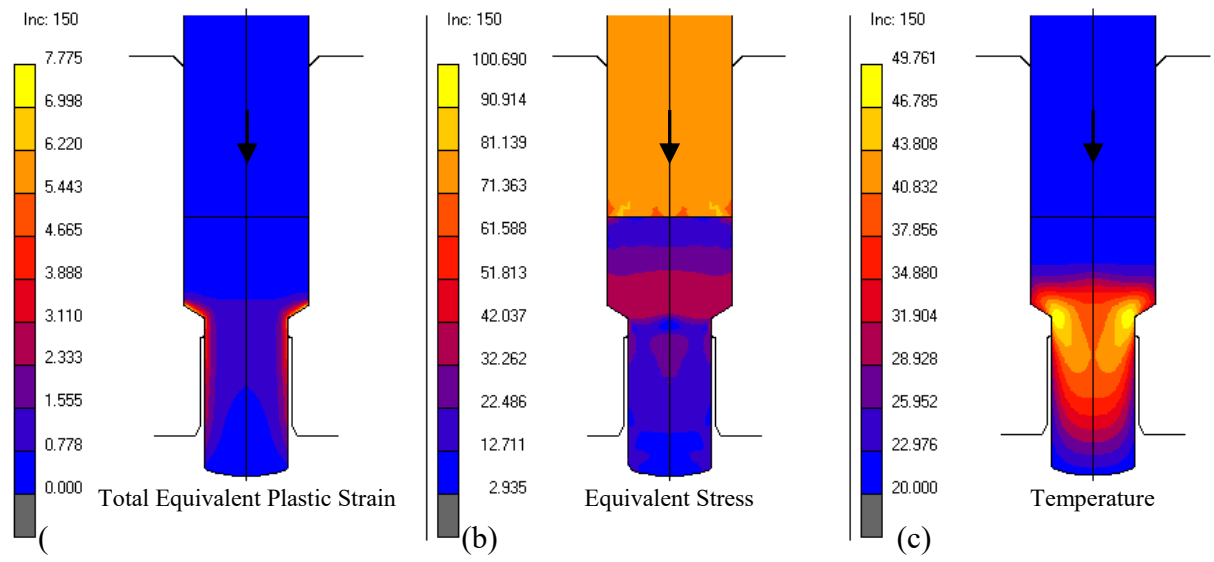

Fig. 2. Variation fields of parameters: (a) strain; (b) stress [MPa]; (c) temperature $\left[{ }^{\circ} \mathrm{C}\right]$.

Figure 2 shows the flow of the material through the die, the non-uniform deformation in the volume of the workpiece (the maximum outside of the extruded part) and the significant increase of the material temperature due to the thermal effect of the deformation.

Next, equivalent stresses from forward extrusion dies were analyzed, for various values deformation cone angle of the die in order to optimize the process. Three different cases of dimensions of the deformation cone of die have been chosen to be analyzed (Table 2).

Table 2. The cases studied for the cone die angle.

\begin{tabular}{|c|c|c|c|}
\hline $\begin{array}{c}\text { Cone angle } \\
\text { [degrees] }\end{array}$ & Case I & Case II & Case III \\
\hline$\alpha$ & $45^{\circ}$ & $60^{\circ}$ & $90^{\circ}$ \\
\hline
\end{tabular}
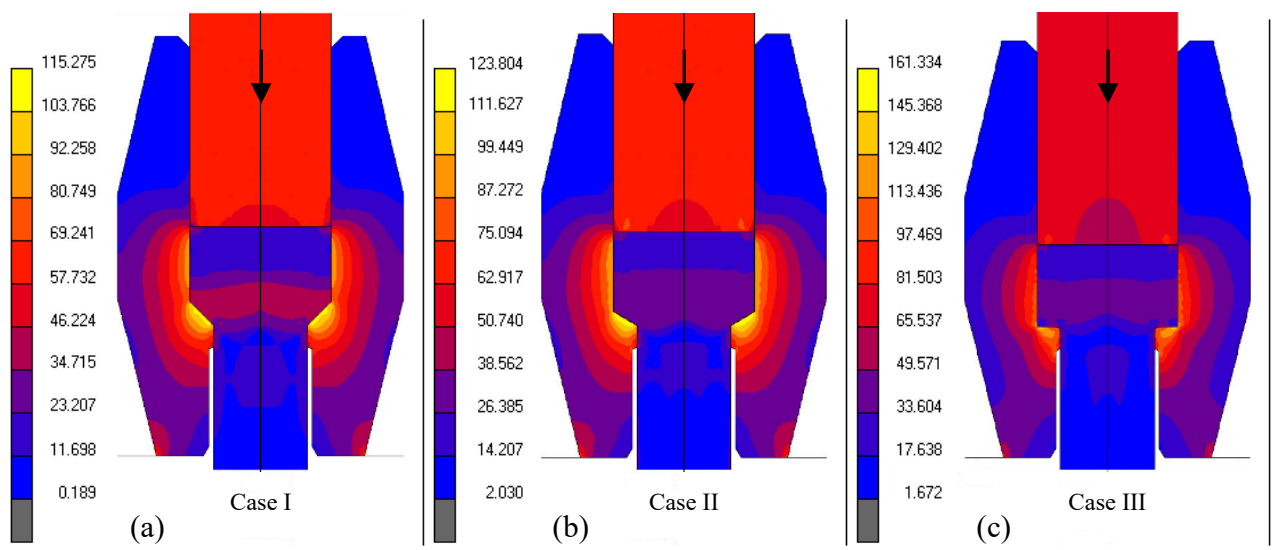

Fig. 3. Variation field of equivalent stress [MPa] from dies, in all the three studied cases. 
In Figure 3 can be observed that stresses have maximum values in the deformation cone of the dies, but do not exceed the allowable strength at break of the steel from which dies are made (15CrNi6). The stresses from the dies increase as the angle of the deformation cone of die gets wider and the highest value is reached at the die with angle $\alpha=90^{\circ}$ (Figure 3.c).

An analysis of the data obtained for the three simulated cases can be done by examining the graph from Figure 4.

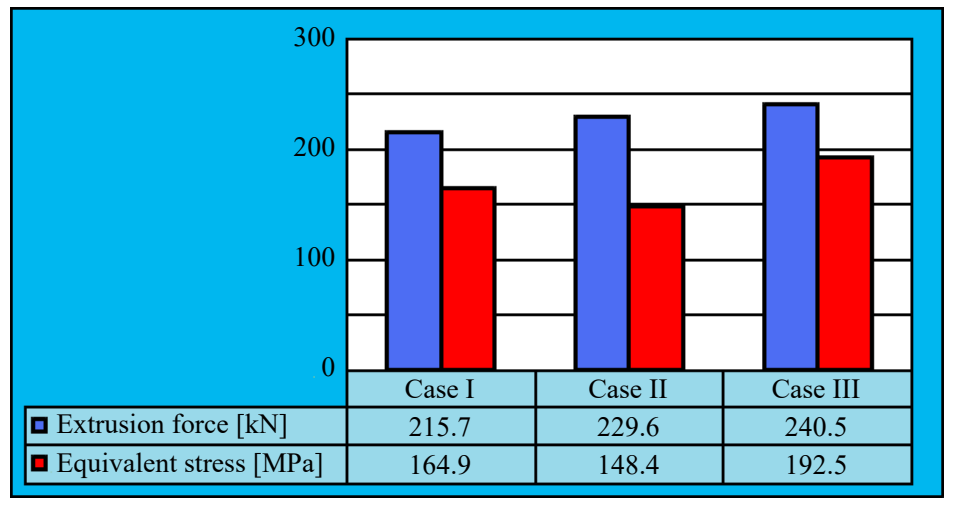

Fig. 4. The maximum values of extrusion forces and stresses from dies in the three cases.

The forward extrusion forces values show that by increasing with one third $\left(15^{\circ}\right)$ the die's deformation cone angle, the force increases by about $6,5 \%$ (case II) and by doubling the value of this angle, extrusion force increases by about $11,5 \%$ (case III). Maximum equivalent stresses of dies, compared to the case I which is considered a reference, decrease by about $10 \%$ in case II and increase by about $17 \%$ in case III.

After obtaining finite element model it is important to check the agreement between this and the experimental model that it represents. Verification is required for validation of the model obtained and this is made by comparing the some parameters obtained by measuring from experiments, with values of the same parameters determined by simulation [13].

Three dies with different deformation cone angles, a punch, nine workpieces with the dimensions $\varnothing 30 \times 40 \mathrm{~mm}$ and a hydraulic press of $75 \mathrm{tf}$ were used to perform the tests. Three experiments were planned and performed for each case. The data acquired from the extrusion process was: extrusion force $[\mathrm{kN}]$, extrusion pressure $[\mathrm{MPa}]$ and press ram stroke $[\mathrm{mm}]$. The experimental system used and some parts obtained are shown in Figure 5.a.
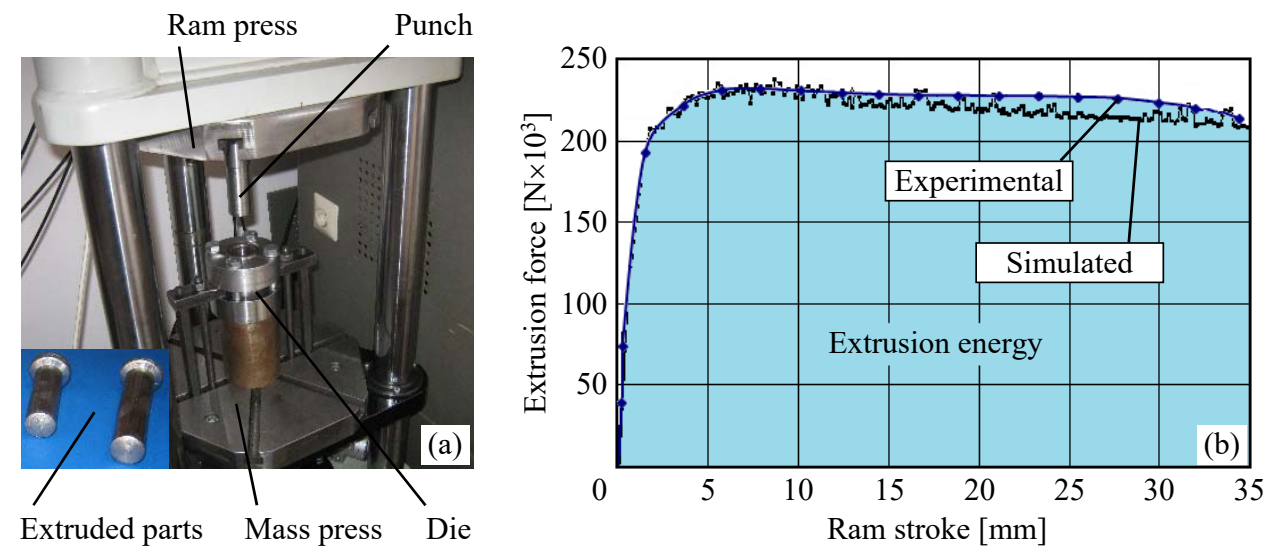

Fig. 5. Experimental extrusion system (a) and extrusion force vs. ram stroke for case II (b). 
Experimental data was acquired from the transducers with which the hydraulic press is equipped through a National Instruments acquisition board and a laptop. The validation of the finite element model has been made by comparing the numerical data obtained by simulation with experimental data for extrusion force. Experimental and simulated curves for extrusion force vs. press ram stroke show a good agreement between the numerical data and those from the experiments (Figure 5.b). In finite element simulation, the compatibility between material model, physical model and type of analysis is very important. If finite element simulation is performed with the appropriate material model, physical model and type of analysis, results can be obtained with sufficient precision for the simulation to be considered correct [14].The results presented in Figure 5 show that the finite element model developed provide accurate data for numerical simulation performed in this study.

\section{Conclusions}

The analysis of cold forward extrusion process shows a good agreement between the data obtained by means of the developed finite element model and data acquired from experiment. Thus, extrusion force values extracted from finite element simulation results show a deviation of about $10 \%$ from the extrusion force recorded in experiment. The main conclusions of this study are: (1) Equivalent stresses from extrusion tools vary directly with deformation cone angle of the die; (2) Equivalent elastic strains in the dies, for all three cases considered are low, below $1 \mu \mathrm{m}$; it shows that the dies were well designed, are sufficiently rigid and provide the necessary precision for the extruded part; (3) Forward extrusion force increases with the growth of die's deformation cone angle; (4) The stresses in the dies reach a minimum value in the case II $\left(\alpha=60^{\circ}\right)$, which suggest that it exists an optimum value of the die's deformation cone angle value, for which the dies' breaking risk is minimal.

\section{References}

1. H. Zhang, X. Li, X. Deng, A.P. Reynolds, M.A. Sutton, J. Mater. Process. Technol., 253, 17 (2018)

2. K. Pieła, M. Wróbel, K. Sztwiertnia, M. Jaskowski, J. Kawałko, M. Bieda, M. Kiper, A. Jarzębska, Mater. Des., 117, 111 (2017)

3. S.S. Jamali, G. Faraji, K. Abrinia, Mater. Sci. Eng. A, 666, 176 (2016)

4. M. Haase, A.E. Tekkaya, J. Mater. Process. Technol., 217, 356 (2015)

5. T. Tang, Y. Shao, D. Li, L. Peng, Y. Peng, S. Zhang, P. Wu, J. Alloys Comp., 730, 161 (2018)

6. P. Karami, K. Abrinia, Int. J. Mech. Sci., 74, 112 (2013)

7. M. Saboori, M. Bakhshi-Jooybari, M. Noorani-Azad, A. Gorji, J. Mater. Process. Technol., 177, 612 (2006)

8. C. Hu, Q. Yin, Z. Zhao, J. Mater. Process. Technol., 249, 57 (2017)

9. A. Farhoumand, R. Ebrahimi, Progr. Nat. Sci.: Mat. Int., 26, 650 (2016)

10. V. Jayaseelan, K. Kalaichelvan, S.V. Ananth, Procedia Eng., 97, 166 (2014)

11. S. Hojda, K.J.X. Sturm, M. Terhorst, F. Klocke, G. Hirt, Procedia Eng., 207, 437 (2017)

12. M.A. Mihalache, G. Nagîţ, M.I. Rîpanu, M. Boca, I.I. Hurja, Appl. Mech. Mater., 657, 725 (2014)

13. D. Luca, MATEC Web of Conf., 112, 02008 (2017)

14. D. Luca, Trans. Nonferrous Met. Soc. China, 25, 2331 (2015) 